\title{
La gerencia universitaria e Internet
}

Rosa María Salom*

\section{Resumen}

La incorporación de las nuevas tecnologias en la gerencia universitaria es analizada a partir de sus páginas web. Se verifica la existencia de infraestructuras tecnológicas y sus diversas aplicaciones en actividades de investigación, labores informativas, toma de decisiones y búsqueda de la integración institucional.

Palabras clave: Comunicación, gerencia, nuevas tecnologías, universidad, información, internet.

\section{University Management and Internet}

\section{Abstract}

The incorporation of new technologies in university management is analyzed on the basis of Web-pages. The existence of technological infrastructures and their diverse applications in research activities, information gathering, decision making, and the search for institutional integration, are verified.

Key words: Comunication, Management, New technology, University, Information, Internet.

\section{Recibido: 27-02-98 . Aceptado: 29-05-98}

* Licenciada en Comunicación Social, Mención Periodismo Audiovisual. Master in Arts (Radio \& Television) y Master in European Business Communication. Profesora de la Escuela de Comunicación Social y de la Maestría en Ciencias de la Comunicación y la Información de la Universidad del Zulia. Coordinadora del Área de Nuevas Tecnologias del Centro de Investigaciones de la Comunicación y la Información 


\section{Introducción}

Las últimas décadas han sido testigos de un extraordinario interés en los conceptos y consecuencias de la Sociedad de la Información y por ende, en los profundos cambios que se están verificando en aspectos políticos, sociales, económicos, tecnológicos e institucionales.

La complejidad del cambio es enorme y envuelve a todos los sectores. Resulta difícil determinar de manera certera, qué porcentaje de este cambio es producto de la evolución tecnológica y si se trata de un fenómeno separado que ha coincidido en el tiempo; lo importante es que se ha producido una evolución y modelos anteriores están quedando obsoletos y se transforman a gran velocidad.

El enorme atractivo que ofrece el sector de las telecomunicaciones está basado en la necesidad, reconocida a escala mundial, de contar con una adecuada infraestructura de comunicaciones que posibilite el desarrollo.

Por qué esta preocupación por las comunicaciones? No faltará quien opine que se trata de una moda pasajera. Tal parece que no. Autores como Annie Bartoli (1992) plantean que la comunicación debe ser un medio de motivación de estrategia e incluso de desempeño y concluye que la empresa u organización no tiene opción pues, a pesar de sus límites, la comunicación formal es indispensable tanto en el plano interno como en el externo.

La teoría general de la comunicación establece que cualquier medio o tecnología de las comunicaciones actúa para producir contacto entre personas, al compartir ideas o símbolos. Al decir contacto, nos estamos refiriendo a cualquier relación inmediata, recíproca y evidente, como los contactos cara a cara, aunque también debemos considerar relaciones muy distantes en tiempo y lugar, y unidireccionales como por ejemplo un artículo en el periódico o un programa grabado de televisión.

Las modernas tecnologías de comunicación, especialmente las electrónicas basadas en el computador, buscan incrementar el contacto y, por lo tanto, disminuir la "distancia" entre personas -tanto física, temporal o social- a través del medio de comunicación" (Caldwell, 1993).

Cuando la gente usa sus computadores personales o sus estaciones de trabajo como medio de comunicación, los utillzan para conectarse a través de una red con otros usuarios para intercambiar información e ideas dentro de un ambiente social e intelectual del medio (Greller, 1993).

Internet es un buen ejemplo de cómo un computador puede ser utilizado como medio de comunicación. Como sabemos, internet es una red de redes que interconecta universidades, empresas comerciales, organizaciones no gubernamentales, bibliotecas, servicios públicos, etc., a miles de computadores (o usuarios) que participan de la denominada "aldea global" (Pasquali, 1998).

Algunos investigadores (Hafner y Markoff, 1991; Schoffstall, 1991) estiman el crecimiento de usuarios de Internet (universidades, investigadores de pro- 
yectos e instituciones militares) entre dos a tres millones diarios.

Si el propósito de utilizar un Sistema de Comunicación basado en Computadores es aumentar la productividad, debe asegurarse una perfecta adecuación entre el sistema y las necesidades de la organización. Esto permitirá los tipos y modos deseados de comunicación, con un mínimo de restricciones percibidas y un mínimo de costos organizacionales o económicos. (Eason, 1988).

La Asociación Española para el Desarrollo de la Función Social de las Comunicaciones (FUNDESCO), indica que los rápidos desarrollos tecnológicos están permitiendo aumentar la velocidad y capacidad de transmisión de los equipos de telecomunicaciones y por su parte, los clientes o usuarios están adoptando una posición activa en la demanda de servi- cios de telecomunicaciones, servicios cada vez más sofisticados debido a la creciente complejidad de las relaciones económicas mundiales.(1994: 11-14).

\section{¿Qulénes están en Internet?}

Aunque la distribución mundial de universidades con páginas web es desigual, en promedio, " $31,4 \%$ de las organizaciones de educación superior en el mundo poseen una presencia en Internet, entre las cuales $74,7 \%$ son universidades y el resto organizaciones no-universitarias de educación superior" (Silvio, 1998:3).

En el gráfico No. 1 se observa que América Latina presenta porcentajes similares a las tendencias mundiales, pero en el caso venezolano, la autora pudo constatar que la casi totalidad de las

Gráfico No. 1

Universidades con Sitio Web

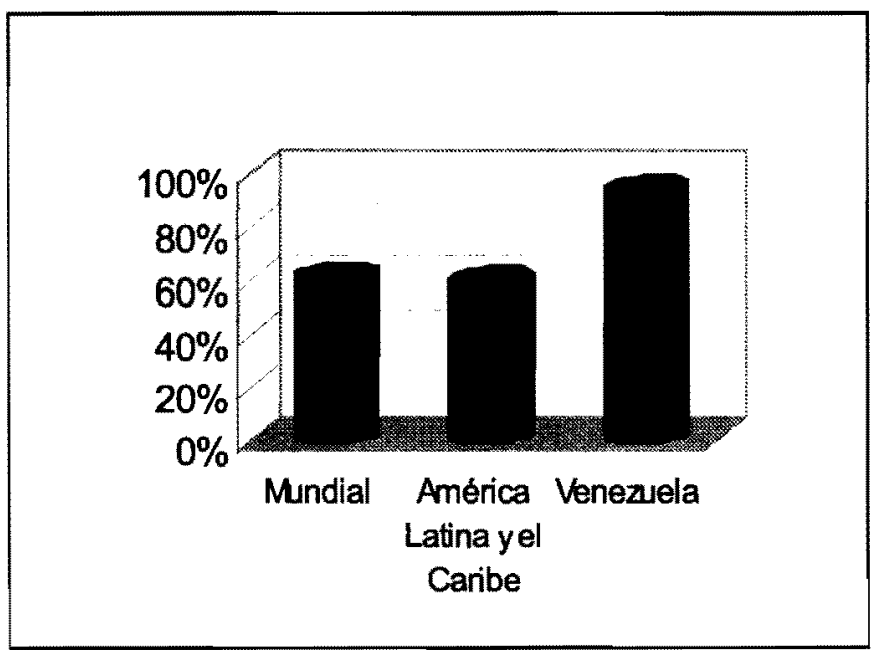

Fuente: Medición propia en base a Josê Silvio "La Virtualización de las Universidades". 
universidades públicas y un alto porcentaje de las privadas tienen su propio sitio en el Web (ver gráfico No. 2). Pero, como señala Silvio (1998:4): "Lo importante es qué tipo de presencia tienen y tendrán las universidades en Internet y que uso harán de sus centros de servicio informativo y sitios web".

Las crecientes presiones de los sectores público y privado sobre las universidades públicas venezolanas a fin de que éstas se tornen más eficientes, han hecho imperativa la revisión profunda de sus sistemas operativos, dentro de los cuales, el de las comunicaciones es fundamental.

No sorprende entonces que la gran mayoría de las universidades públicas venezolanas haya respondido, cada una a su manera, a los requerimientos de actualización de sus sistemas de informa- ción académica, administrativa y de apoyo a la investigación; casi todas ellas, han comenzado a instrumentar sistemas de automatización de la información y la gran mayoría posee una página WEB en Internet que refleja en mayor o menor grado, los esfuerzos de cada institución en esta materia.

\begin{abstract}
En el cumplimiento de su misión, la universidad moderna funciona (o deberia funcionar) como una gran organización cuya cohesión interna y coherencia con su entorno se encuentran garantizadas por la calidad de sus procesos informacionales. (Universidad Simón Bolivar, st: 2).
\end{abstract}

A pesar de que la gran mayoría de las universidades públicas venezolanas poseen un sitio web, no todas han incor-

Gráfico No. 2

Presencia en el Web de Universidades Venezolanas

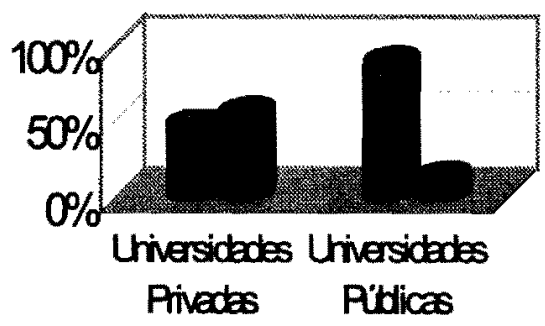

IINO

Fuente: Elaboración propia en base a José Silvio "La virtualización de las Universidades 
porado las nuevas tecnologías a sus sistemas gerenciales.

La tabla No. 1 nos permite confirmar que si bien el $89 \%$ de las universidades nacionales poseen una página web, utilizada en un $100 \%$ de los casos para informar, es también cierto que algo más de la mitad las emplea para lograr la integración institucional, el $47 \%$ de las universidades como apoyo a la investigación, mientras que sólo un $20 \%$ lo incorpora en la toma de decisiones.

Las universidades Central de Venezuela, Simón Bolívar, del Zulia, de Los Andes, Carabobo y la Experimental Simón Rodríguez, parecen ser las casas de estudios superiores que hacen un uso más amplio de las nuevas tecnologías.

La virtualización (parcial o total) de estas organizaciones puede ser un factor transformador de sus estructuras y funciones, un instrumento para mejorar su cobertura, calidad, pertinencia y equidad de acceso y una manera de construir una nueva identidad en la nueva "sociedad del conocimiento" en proceso de gestación. La viabilidad y factibilidad de convertir a la virtualización en un factor de calidad académica dependerá del enfoque utilizado para implantarla. (Sil. vio, 1998:2).

\section{El ciberespącio Universitario Venezolano}

La Universidad Simón Bolívar inició en 1987 la discusión interna sobre las dis- tinciones entre las industrias de la información e industrias del conocimiento. En este contexto se constituyó en 1989, una Comisión Asesora para la Organización y Desarrollo del Sistema de Información de la Universidad Simón Bolivar (Universidad Simón Bolívar, SF, 2).

Actualmente esta casa de estudios cuenta con un Sistema de Información cuya gerencia reside en la Secretaria de la Universidad. La DGI, Dirección para la Gestión de la Información, es una unidad de calificación técnico-administrativa adscrita a la Secretaría General de la Universidad, creada como estructura de apoyo al Proyecto SI-USB, "Sistema de Información de la Universidad Simón Bolivar" .

La Dirección de Gestión de la Información cuenta con un equipo mínimo de recursos humanos especializados en Computación, Tecnología de la Información y Sistemas de Información que basa su actuación en el esquema de la organización matricial característica de la USB. Esta dirección comparte recursos con otras dependencias involucradas en el área de gestión de la información o con las que agrupan otros recursos especializados. Su gestión sigue la dirección marcada por la misión del CC/SI-USB y sus senicios incluyen la administración de una página WEB con información pertinente no sólo a los servicios y recursos de la universidad, sino también al propio servicio de información.

El propósito global del Sistema de Información de esta universidad no se orienta realmente hacia la optimización

Esta sección se desarrolla básicamente a partir de la información contenida en las respectivaas páginas WEB de las universidades, cuyas direcciones electrónicas se incluyen en las referencias bibliográficas. 


\begin{tabular}{|c|c|c|c|c|c|c|c|c|}
\hline \multicolumn{9}{|c|}{$\begin{array}{l}\text { Tabla N } N^{2} 1 \\
\text { Sistemas Comunicaciones de Universidades Nacionales }\end{array}$} \\
\hline \multicolumn{2}{|c|}{ Institución } & \multicolumn{3}{|c|}{ Tipos de Servicios } & \multicolumn{4}{|c|}{ Objetivos o Funciones } \\
\hline & Tipos de usuario & Televisión & Radio & Electrónico & $\begin{array}{l}\text { Apoyo a la } \\
\text { Invostigación }\end{array}$ & informar & $\begin{array}{c}\text { Toma de } \\
\text { Docisiones }\end{array}$ & \begin{tabular}{|l|} 
Integración \\
Institucional \\
\end{tabular} \\
\hline 1 UCV & $\begin{array}{ll}\text { Interno } & x \\
\text { Externo } & x\end{array}$ & & & $\frac{x}{x}$ & $x$ & $\frac{x}{x}$ & $x$ & $x$ \\
\hline $2 \mathrm{LUZ}$ & $\begin{array}{|ll|}\text { Interno } & x \\
\text { Externo } & x \\
\end{array}$ & $x$ & $\begin{array}{l}x \\
x \\
\end{array}$ & $x$ & $x$ & 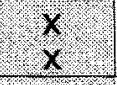 & & $x$ \\
\hline 3 ULA & $\begin{array}{ll}\text { Interno } & x \\
\text { Externo } & x\end{array}$ & $\begin{array}{l}x \\
x \\
\end{array}$ & $\begin{array}{l}x \\
x\end{array}$ & $\begin{array}{l}x \\
x\end{array}$ & $\begin{array}{l}x \\
x\end{array}$ & $\begin{array}{l}x \\
x\end{array}$ & & $\begin{array}{l}x \\
x\end{array}$ \\
\hline 4 USB & \begin{tabular}{ll|} 
Interno & $x$ \\
Externo & $x$ \\
\end{tabular} & $x$ & & $\frac{x}{x}$ & $x$ & $\begin{array}{l}x \\
x \\
x\end{array}$ & $x$ & $x$ \\
\hline 5 UDO & $\begin{array}{l}\text { Interno } x \\
\text { Externo } x \\
\end{array}$ & & & $\begin{array}{l}x \\
x\end{array}$ & & $\begin{array}{l}x \\
x\end{array}$ & & $x$ \\
\hline $6 \cup C$ & $\begin{array}{ll}\text { Interno } & x \\
\text { Externo } & x\end{array}$ & & $\begin{array}{l}x \\
x\end{array}$ & $x$ & $x$ & $x$ & & $x$ \\
\hline 7 UNA & $\begin{array}{l}\text { Interno } x \\
\text { Externo }\end{array}$ & & & $x$ & & $x$ & & \\
\hline 8 UCLA & $\begin{array}{ll}\text { Interno } & x \\
\text { Externo } & x \\
\end{array}$ & & & $\frac{x}{x}$ & & $\begin{array}{l}x \\
x\end{array}$ & & \\
\hline 9 UNELLEZ & $\begin{array}{ll}\text { Interno } & x \\
\text { Externo } & x\end{array}$ & & & $\begin{array}{l}x \\
x \\
\end{array}$ & & $\begin{array}{l}x \\
x\end{array}$ & & $x$ \\
\hline 10 UNESR & $\begin{array}{ll}\text { Interno } & x \\
\text { Externo } & x\end{array}$ & & & $\begin{array}{l}x \\
x \\
\end{array}$ & $x$ & $x$ & $x$ & $x$ \\
\hline 11 UNET & $\begin{array}{ll}\text { Interno } & \mathrm{x} \\
\text { Externo } & \mathrm{x}\end{array}$ & & & $\begin{array}{l}x \\
x \\
\end{array}$ & & $\begin{array}{l}x \\
x\end{array}$ & & \\
\hline 12 UNEXPO & $\begin{array}{ll}\text { Interno } & x \\
\text { Externo } & x\end{array}$ & & & $\begin{array}{l}x \\
x\end{array}$ & & $x$ & & \\
\hline 13 UNERMB & $\begin{array}{ll}\text { Interno } & x \\
\text { Externo } & x \\
\end{array}$ & & & $x$ & & X. & & \\
\hline 14 UNEFM & $\begin{array}{ll}\text { Interno } & x \\
\text { Externo } & x \\
\end{array}$ & & & $x$ & $x$ & $\begin{array}{l}x \\
x\end{array}$ & & \\
\hline 15 UNEG & $\begin{array}{ll}\text { Interno } & x \\
\text { Externo } & x\end{array}$ & & & $\begin{array}{l}x \\
x\end{array}$ & & $x$ & & $x$ \\
\hline 16 UNERG & $\begin{array}{l}\text { Interno } \\
\text { Externo }\end{array}$ & & & & & 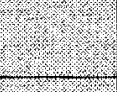 & & \\
\hline 17 UNEP & $\begin{array}{l}\text { Interno } \\
\text { Externo } \\
\end{array}$ & & & & & 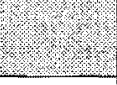 & & \\
\hline $100 \%$ & & & & $89 \%$ & $47 \%$ & $100 \%$ & $20 \%$ & $60 \%$ \\
\hline
\end{tabular}


de las funciones que caracterizan o definen a un ambiente, ". sino hacia los individuos que ejecutan tales funciones, es decir, al mejoramiento de sus capacidades de utilización de la tecnología informática y de la información". Se expresa claramente el propósito de incrementar la productividad, el "nivel de satisfacción en el trabajo y la capacidad de ajuste individual y grupal a cambios organizacionales de crecimiento y competencia".

El Rectorado de la Universidad Central de Venezuela, interesado en mejorar cualitativa y cuantitativamente las actividades de generación de conocimientos mediante la investigación científica, humanistica y tecnológica creó el Sistema de Apoyo Gerencial a la Investigación, con la idea de "superar un conjunto de deficiencias actuaimente existentes, y satisfacer necesidades sentidas que no han tenido respuestas institucionales hasta el presente".

El proyecto de la UCV, actualmente en fase de implementación, incluirá una estructura organizativa del SAGI-UCV que contendría, al menos, las siguientes cuatro unidades:

1) Unidad de Información e Inteligencia

2) Unidad de Capacitación de Recursos Humanos

3) Unidad de Asesoría y Servicios

4) Unidad de Apoyo Logístico

Tal como se indica en su propia página WEB:

La creación del Sistema de Apoyo Gerencial a la Investigación en la Universidad Central de Venezuela (SAGIUCV) se sustenta en la concepción de gestión competitiva de Centros de Investigación, dentro de la cual se re- conocen cinco funciones claves para el adecuado desempeño de una organización de excelencia.

Como se observará, la aproximación de la UCV incorpora una visión gerencial de la institución, desconocida en las universidades públicas venezolanas hace menos de dos décadas. La declaración de principios de esta universidad revela un apoyo a las estructuras que propugnen activamente "el cambio de paradigmas" para lograr "mayor productividad", y plantea que su desempeño "_debe ser anticipativo, integral y sistémico, debe reconocer la necesidad de garantizar la calidad integral de su "producto" y de propender hacia la suficiencia económica de la institución". Esta visión plantea el deseo de "...crear capacidades propias en las funciones críticas de la gerencia, como instrumento para apoyar la investigación".

La respuesta de la Universidad de los Andes a estos retos ha sido sistemática. Además del diseño e implementación de una estación de televisión (TVE-ULA) y de tres estaciones de radio, esta casa de estudios creó dos servicios de información automatizados: el SeDULA (Servicio de Documentación de la Universidad de Los Andes) y el SIGULA (Sistema de Información Gerencial).

El principal objetivo de SeDULA es reunir y sintetizar información ya procesada, proveniente de los entes centralizados y las diferentes dependencias universitarias, con el fin de "ofrecer información relevante, pertinente y oportuna de la Universidad de Los Andes a la comunidad regional, nacional e internacional". Para ello se apoya en los servicios de la 
Red Local (RedULA) y la Red Global INTERNET, a través del sistema World Wide WEB. Como se observará, esta concepción de la ULA, incorpora la visión corporativa de la universidad al distinguir entre las necesidades de sus públicos internos y externos. Con este servicio se pretende establecer un esquema general que facilite organizar la información administrativa y académica, para incorporar el resto de la información institucional que pretenda ser incorporada a estos servicios por otras dependencias universitarias.

El objetivo principal del Proyecto SiGULA es suministrar a la gerencia de la ULA los indicadores necesarios que sirvan de apoyo a la toma de decisiones y permitan "sustentar las discusiones acerca del ser y deber ser de la institución llevadas a cabo en estos niveles estratégicos". Se persigue a su vez, que la información contenida en la base de datos del SIGULA alimente buena parte de la información documental incorporada a Internet mediante el proyecto SeDULA.

El tema de la comunicación ha sido protagonista del debate universitario en LUZ desde hace más de dos décadas. La creación de instancias responsables de las comunicaciones internas y externas (Oficina de Prensa, Departamento de Relaciones Públicas, Dirección de Comunicaciones), los esfuerzos por la definición y aplicación de Políticas Comunicacionales y la posición de vanguardia en el diseño e implementación de sofisticadas tecnologías como el Centro de Televisión Educativa, la emisora LUZ-FM 102.9, el cableado con fibra óptica del campus universitario y los servicios de acceso a Internet son algunas muestras de la cons- tante preocupación de las autoridades de LUZ por las comunicaciones (Villalobos, 1998).

Pareciera que los esfuerzos de la Universidad del Zulia se enfilaran hacia un propósito coherente. Recientemente se dio a conocer a la opinión pública la declaración de Filosofía de Gestión de la Universidad del Zulia, la cual plantea como una de las estrategias institucionales " poner en práctica un Sistema de Información Gerencial oportuno, preciso y clasificado según las necesidades de información por niveles que permita una efectiva toma de decisiones".(1994:2223) El mismo documento señala dentro de las políticas de apoyo académico, la necesidad de:

Desarrollar y mantener sistemas automatizados de información, adecuados a las necesidades de la Universidad en su conjunto, que permitan la toma de decisiones oportunas y racionales y ...Desarrollar y perfeccionar mecanismos de comunicación e información que permitan consolidar la presencia de la institución en la región y el país. (LUZ, 1994: 29).

De hecho, la infraestructura de telecomunicaciones de LUZ es una de las más sofisticadas del país dentro del subsector de la educación superior.

- Mantiene un sistema de interconexión por cable coaxial;

- posee un sistema de comunicación por radio para interconectarse con los núcleos de Punto Fijo (Estado Falcón) y Cabimas, 
en la Costa Oriental del Lago de Maracaibo;

- desde 1991, opera una emisora en frecuencia modulada estéreo, LUZ-FM 102.9, la cual tiene una cobertura de $5 \mathrm{Khz}$ que lleva el mensaje universitario hasta los estados Falcón, Trujillo y Mérida;

- su Centro de Televisión Educativa (activo desde 1975) transmitirá 10 horas de programación, vía cable a toda la ciudad a partir del mes de septiembre y permitirá la comunicación televisiva desde las facultades hacia el resto del campus en circuito cerrado;

- cuenta con un sistema de Servicios Bibliotecarios conectados en línea desde cada una de las facultades con la Unidad Central, y de alli, a través del Instituto de Cálculo Aplicado (ICA), se interconecta con el Conicit y la Universidad de los Andes, para servir a todas las universidades y centros de investigación del país;

- está concluyendo un cableado por fibra optica que actuará como carrier para las señales de televisión, telefonía y de servicios de información en línea.

En cuanto a la Universidad de $\mathrm{Ca}$ rabobo desde Mayo de 1987, funciona la Fundación Centro de Información y Do. cumentación, CID, cuyo objetivo es "planificar, desarrollar, crear y administrar los servicios de información que mejor sirvan al desarrollo de la ciencia, la investiga- ción, la tecnología, la educación, la extensión y la gestión".

EI CID se perfila como la gran Biblioteca Virtual de la Región Central del Pais. Se trata de una institución que desarrolla actividades relacionadas con la gerencia de la Intormación. Al igual que en otras universidades de país, se consideran las necesidades de información de sus públicos interno y externo: Tiene como objetivo principal establecer mecanismos adecuados que permitan con agilidad el acceso y difusión de intormación cientifica, tecnológica, social y humanística a usuarios de la comunidad universitaria, así como extrauniversitaria de la región y del pais en general.

REDISA es la Red de Información y Servicios Académicos de la Universidad Centro-Occidental Lisandro Alvarado (UCLA), la cual tiene como objetivo principal ofrecer a la comunidad universitaria y público en general un medio informativo electrónico de las actividades que se están llevando a cabo en las diferentes unidades académicas de esa institución. Esta red está siendo estructurada por un grupo de profesionales del área de Informática conjuntamente con los directores de cada una de las dependencias académicas. El proyecto comenzó en el mes de Diciembre de 1997 y actualmente que están organizando la información que presenta por Internet.

La Universidad Simón Rodríguez creó la Dirección General de Sistemas de Información que coordina el Proyecto de Red de Interconexión Universitaria, el cual incluye tres áreas básicas con los objetivos de informar a los públicos internos y externos, interconectar los diversos núcleos, automatizar los sistemas admi- 
nistrativos y académicos e innovar los sistemas de educación a distancia.

La Universidad ha adquirido los equipos y desarrollado aplicaciones computarizadas para agilizar la descentralización de las funciones de registro de nómina, registro y control presupuestario, administración financiera y contable, inventario de bienes y adquisición de bienes muebles, utilizando para ello, una red automatizada que permite la carga $y$ transferencia inmediata de la información, desde las unidades desconcentradas vía tele-procesos hasta las unidades centrales.

El resto de las de las universidades públicas (con la excepción de dos universidades experimentales), también tiene presencia en el web, y un sistema de información en línea, aunque menos sofisticado que las universidades mencionadas previamente.

Dentro de este segundo grupo de universidades resulta pertinente mencionar el esfuerzo realizado por la Universidad de los Llanos Ezequiel Zamora (UNELLEZ) que viene utilizando su página WEB como un mecanismo para integrar los diversos núcleos que la componen: Barinas, Apure, Cojedes, y Guárico.

Esta universidad determinó la necesidad de que sus acciones se orienten hacia un efectivo aprovechamiento de las oportunidades existentes "a través del establecimiento de nuevas formas de relación entre los distintos actores de la sociedad: Gobierno, Instituciones, Gremios y Comunidades para el logro de los fines planteados".
En este sentido, creó una oficina de enlace, establecida en la ciudad de Caracas, cuyos objetivos son:

- Promover y mantener el intercambio técnico-científico, social, humanistico y cultural con el resto de Instituciones que integran el Sub-sistema Nacional de Educación Superior, especialmente con las demás Universidades Nacionales, públicas y privadas.

- Establecer y mantener relaciones con organismos internacionales.

- Informar a los niveles académicos sobre oportunidades de intercambio y desarrollo profesional que ofrezcan organismos nacionales y extranjeros.

- Establecer relaciones con organismos nacionales o internacionales para el logro de financiamiento a programas académicos y administrativos.

- Facilitar la gestión administrativa y financiera de la Unellez ante instituciones de la administración publica central y descentralizada.

- Promover la imagen de la Unellez a escala nacional e internacional.

Paralelamente, la UNELLEZ estableció un convenio con la Fundación de Información y Documentación de la Universidad de Carabobo para el establecimiento de un servicio especializado de consulta personalizada en sala, que permite a sus diversos núcleos el acceso a las bases de datos disponibles, así como 
el servicio de consulta en línea por medio de terminales remotos vía modem.

La Universidad Nacional Experimental del Táchira ha dado inicio a un ambicioso proyecto de cableado con fibra optica que permitirá prestar un servicio de redes al sector administrativo y académico, y coordinará los procesos vinculados con el inicio y desarrollo de los sistemas de información académica: Decanatos, Biblioteca, Centro de Información Regional, Centros y Laboratorios de Investigación, y demás dependencias que requieran el desarrollo de sistemas de informa. ción.

El proyecto de la UNET es el resultado de una asociación con la empresa IBM de Venezuela y consiste en la instalación y puesta en marcha de 450 puntos de red de alta velocidad mediante una troncal de fibras ópticas.

Las restantes universidades que han colocado centros información en Internet son la Universidad Nacional Experimental Francisco de Miranda, la Universidad Nacional Abierta, la Universidad de Oriente, la Universidad Nacional Experimental Politécnica Antonio José de Sucre, la Experimental Rafael Maria Baralt y la Universidad Tecnológica del Centro.

Estas universidades, con un presupuesto menor (CNU-OPSU, 1998: 118) y en general, de más reciente creación poseen al menos una página web informativa que incorpora los programas de estudio y servicios ofrecidos. En general, estas universidades no cuentan con servicios de correo electrónico entre los miembros del personal, automatización de procesos de inscripción, consulta de base de datos en línea, servicios bibliotecarios, etc.

\section{La respuesta del sector privado de la educación superior}

El reto que significa mejorar las comunicaciones institucionales a través del uso de nuevas tecnologías ha sido enfrentado también por las universidades privadas. Varias de ellas tienen su propia página en el WEB: la Universidad Metropolitana, la Universidad Católica Andrés Bello, la Universidad de Nueva Esparta, la Universidad Rafael Belloso Chacín (cuya página web está actualmente fuera de servicio), y la Universidad Rafael Urdaneta.

Otras universidades privadas como la Santa María y la Bicentenaria de Aragua, poseen páginas no oficiales, colocadas en el web por sus estudiantes.

En general, no se observó en el análisis de sus páginas web, información que permitiese concluir si las nuevas tecnologias son utilizadas con propósitos diferentes a los informativos.

\section{Reacciun}

Dentro de esta revisión ( $y$ aunque no pertenece a ninguna universidad en particular), debe hacerse una mención especial a Reacciun (Red Académica de Centros de Investigación y Universidades Nacionales). Su página WEB es quizás una de las más informativas del cyberespacio nacional en relación a sus objetivos, misión, visión, y organización. Enuncian su voluntad de erigirse en la "organización líder en Tecnologías de la Información y Comunicación que promueva su uso, en forma creativa, en actividades educativas y de investigación y desarro- 
lio". Resulta notable que nuevamente nos encontramos con el propósito expresado de generar mayores niveles de productividad, competitividad y bienestar.

La definición de su misión nos permite acercarnos aún más a su filosofia:

Interconectar, eficientemente, a través de la telemática y por medio del intercambio y difusión de información a las organizaciones educativas, cientificas y tecnológicas entre sí y con el resto del mundo, promoviendo activamente la creación e intercambio de valor agregado, el desarrollo de actividades educativas y de formación y las de Investigación y Desarrollo para de esta forma incentivar la cooperación entre ellas y potenciar sus capacidades, poniéndolas al servicio de la sociedad.

El origen de esta red se remonta a 1981, cuando el Conicit (Consejo Nacional de Ciencia y Tecnología) creó el Sistema Automatizado de Información Científica y Tecnológica, Saicyt, a fin de atender las necesidades de información de la comunidad científica y tecnológica.

Algunos de los servicios de Internet que ofrece la red son:

- Correo electrónico: Consiste en el servicio de intercambio de correspondencia entre usuarios con acceso a los computadores de la red.

- Transmisión electrónica de mensajes. Incluye el envio automático de un mismo mensaje a múltiples direcciones a la vez.
- Transferencia de archivos: Transmisión electrónica de archivos entre usuarios. Carga, por parte de los usuarios, de archivos bajados de la red.

- Computación remota: Posibilidad de ejecutar programas en computadores ubicados geográficamente distantes al usuario.

- Sesiones interactivas: Comunicación interactiva en línea entre dos o más internautas (talks y chats)

- Gopher y World Wide WEB: Acceso a estos dos protocolos de navegación por Internet.

- Servicio de noticias: Net News. Boletines de informaciones $y$ noticias en-línea. Diferentes tópicos de interés.

Los servicios de información disponibles en Reacciun incluyen todos los servicios de valor agregado creados y por crearse: Foros, Discusiones, Bases de datos de Venezuela y del exterior, Catálogos de las principales bibliotecas del país, Directorios de especialistas, Servicio de recuperación de documentos, Asesoria de los productores de información sobre el desarrollo de productos accesibles en línea, Normas utilizadas nacional e internacionalmente para estos desarrollos, Protocolos, interfaces, lenguajes y paquetes, Especificación de base de datos, Estructura de costos de estos servicios; Otros servicios de interés para la comunidad de usuarios 


\section{Los otros sites}

Si bien Reacciun es el vínculo entre la gran mayoria de universidades y servicios para el sector de la educación superior, existe toda una red de páginas web que proveen información valiosa acerca del quehacer universitario en todas sus manifestaciones.

El cibernauta interesado hallará páginas de los centros de investigación de las universidades nacionales como CeCALCULA, Centro de Cálculo Científico Universidad De Los Andes. Se trata de una página producida y mantenida por la Universidad de Los Andes, el Conicit y la Corporación Parque Tecnológico de Mérida para desarrollar servicios y entrenamiento de personal en el área de Supercomputación y sus aplicaciones.

El Centro de Investigación Aplicada en Sistemas de Información Gerenciada de la Universidad Central de Venezuela tiene una página que puede ser consultada; e igual ocurre con el Programa de Investigación de la Universidad Rafael María Baralt, el cual tiene un site que tiene por función promover, estimular, coordinar y financiar la investigación en esa casa de estudios.

Por otra parte, diferentes laboratorios, escuelas y facultades de diversas universidades han colocado su propia página en el ciberespacio. Navegando a través del link de Reacciun, Eureka, encontramos los vínculos con las páginas de la Facultad de Ciencias Económicas y Sociales y la del Área de Estudios de Postgrado de la Universidad de Carabobo. También hallamos las páginas de las facultades de Ingeniería y Derecho de la Universidad Católica Andrés Bello.
La Universidad del Zulia ofrece las páginas de la Licenciatura en Computación, de la Facultad de Ciencias de LUZ (CIENS) y las del Centro Experimental de Estudios Latinoamericanos (CEELA).

De la Universidad Nacional Abierta encontramos la página de la Mención Matemáticas de la Escuela de Educación, cuyo propósito es formar docentes de matemáticas para la Tercera Etapa de la Educación Básica y la Educación Media Diversificada y Profesional. El Postgrado de esta universidad también tiene su propia página que puede ser consultada .

La Universidad de Nueva Esparta es quizá una de las que posee mayor presencia en el WEB. Alli encontramos las páginas de sus Escuelas de Diseño, Ingeniería Electrónica, de Administración de Empresas Turísticas, Administración, Computación, e Ingeniería Civil, Mención Mantenimiento.

La Facultad de Ingeniería de la Universidad de los Andes, es sin duda alguna, miembro del equipo de fundadores del ciberespacio nacional. Su página WEB es familiar a los investigadores $y$ académicos de todo el país. De esa misma universidad puede ser consultada la página del Laboratorio de Anatomía de Maderas, donde se hallará información en el campo de anatomía de maderas y áreas afines, o el Programa de Estudios de Postgrado en Computación, la de las Facultades de Ciencias y Ciencias Forestales y Ambientales.

Perteneciente a la Universidad Central de Venezuela hallamos la página de la Facultad de Medicina; su objetivo es "formar el personal para garantizar la eficiencia del equipo de salud". El Centro de Estudios del Espacio Arquitectónico po- 
see una página, igualmente se pueden consultar las páginas de la facultad de Ingeniería, de la Dirección de Informática, de la Escuela de Fisica. La UCV nos ofrece la página del Centro de Análisis de Imágenes Biomédicas Computarizadas, la página del Departamento de Matemáticas Aplicadas y la de la Escuela de Derecho.

La Facultad de Ingeniería Eléctrica de la Universidad Simón Bolivar puede ser ubicada. De esta universidad también pueden ser consultadas las páginas de diversos laboratorios: el Laboratorio Docente de Computación que presenta información sobre su organización y servicios y la página del Laboratorio de Electrónica del Estado Sólido, el cual está dedicado a la investigación, cumple funciones docentes como apoyo de asignaturas de Ingenieria Electrónica y en la realización de proyectos de grado y tesis de postgrado.

Además de las páginas oficiales, existen numerosas páginas "no oficiales" producidas por las cátedras o grupos de estudiantes. Ejemplo de ello son la página creada por los estudiantes de Informática de la Escuela de Comunicación Social de la Universidad Católica Andrés Bello, UCAB; los estudiantes de esa casa de estudios parecen ser muy activos en esta materia, pues también hallamos la página del Centro de Estudiantes. Los estudiantes de la Universidad Metropolitana han colocado en el WEB un site no oficial; al igual que los alumnos de la Escuela de Ingeniería Industrial de la Universidad Santa María y los de la Universidad Bicentenaria de Aragua.

Estas no son, por supuesto, todas las páginas producidas por la academia venezolana en Internet. El interesado deberá incursionar y realizar su propia búsqueda a través de browsers nacionales y extranjeros que tienen vínculos directos con nuestras universidades.

\section{Conclusiones}

A pesar de la proliferación de páginas web en Internet, las cuales representan un notable esfuerzo por parte de la comunidad universitaria venezolana, persiste la incomunicación.

Los servicios de información, y en general, las comunicaciones internas $y$ externas de las universidades venezolanas, distan mucho de satisfacer los requerimientos de sus comunidades, tanto en calidad, como en cantidad, oportunidad y pertinencia. Uno de los renglones donde estas deficiencias se hacen más palpables, es sin lugar a dudas, el de las bibliotecas, cada vez más sacrificadas en el contexto de progresivas limitaciones financieras.

La gran mayoría de estas instituciones, a pesar de tener una página en Internet que pueden ser visitadas por los cybernautas, no disponen de servicios de información en línea que permitan a profesores, investigadores y estudiantes hacer consultas en línea de las colecciones aimacenadas en sus propias bibliotecas.

Los servicios de correo electrónico han sido objeto de severas críticas tanto en la Universidad Central de Venezuela como en la del Zulia, por la frecuencia de las interrupciones.

En el área administrativa, sólo la Simón Bolivar pudiese exhibir una utilización integral de los nuevos sistemas, aunque la gran mayoria de las universidades 
(tanto públicas como privadas) poseen algún nivel de automatización en sus procesos administrativos. Sólo la UCV y la UCLA anuncian la integración de las nuevas tecnologías a la toma de decisiones en breve tiempo.

Paralelamente se manifiesta una queja generalizada de la inoperancia de los sistemas tradicionales de comunicación entre autoridades, administradores y comunidad en general y en la gran mayoría, el acceso a equipos de telecomunicaciones $\theta$ informática, tales como computadores, fax o simplemente teléfono, está restringido, no sólo por las limitaciones de cada institución, sino por las deficiencias del servicio telefónico nacional.

¿Será la incorporación de las universidades nacionales a Internet una moda pasajera? Es muy pronto para saberlo, pero es indudable que su auge ha provocado una profunda revisión en la concepción y operatividad de los servicios informativos de numerosas universidades venezolanas. Sus beneficios para la comunidad académica son innegables. Pero su profundización es indispensable si se desea que los beneficios sean permanentes y radicales.

Esta profundización implica:

- el estudio serio de las variables que afectan la productividad $y$ eficiencia de las universidades,

- la definición de políticas de comunicación,

- el establecimiento de estrategias acordes con esas políticas,

- el compromiso de las autoridades universitarias con los sistemas de comunicación e infor- mación desarrollados, que se verificará en

- la asignación de recursos necesarios para su implementación.

\section{Referencias bibliográficas}

Bartoli, Annie (1992). Comunicación y Organlzación, Ediciones Paidós, Barcelona.

Caldwell, Barrett y Taha, Lilas (1993). "Starving at the Banquet: Social

Isolation in Electronic Media." Interpersonal Computing and Technology:

An Electronic Journal for the $21^{\text {st }}$ Century, January, Vol 1, \#1, < http: www.helsinki.fi/science/op-

tek/1994/N2/caldwell.txt> [Consulta: 23 de Marzo 1998].

Centro de Investigaciones Aplicadas en Sistemas de Información Gerenciada. Universidad Central de Venezuela. "Página Principal". </http://www.cosmopoima.ing.ucv.ve>, [consulta: 3 de Marzo 1998].

Centro de Cálculo Cientifico. Universidad de Los Andes. "Página Principal". <http://www .cecal.ula.ve>, [consulta: 3 de Febrero 1998].

CNU-OPSU Separata del Boletín Estadistico de Educación superior 1989-1995. Caracas, 1998.

Eason, K. (1988). Information Technology and Organizational Change en

Caldwell, Barrett y Taha, Lilas (1993).

Facultad de Medicina. Universidad Central de Venezuela. "Página Principal". <http://www.rect.ucv.ve/med/index.htm>, [consulta: 4 de Marzo 1998].

FUNDESCO (1994). Telecomunicaciones 1994. Tendencias, Fundación para el Desarrollo de la Función Social de 
las Comunicaciones (Fundesco), Madrid.

Greller, Leonore M. (1993). "Groupware and Interpersonal Text: The Computer as a Medium of Communication". Interpersonal Computing and Technology: An Electronic Journal for the $21^{\text {st }}$ Century, April, Vol 1, \# 2.<http://nmw. helsinki.fi/science/optek/1994/N2/ grelle .txts [ consulta: 23 de Marzo 1998].

Hafner, K., \& Markoff, J. (1991). Cyberpunk: Outlaws and hackers on the computer frontier en GRELLER, Leonore $M$. (1993). "Groupware and Interpersonal Text: The Computer as a Medium of Communication".

Laboratorio de Electrónica del Estado Sólido. Universidad Simón Bolívar. "Página Princlpal". <(http://159.90.166.205/>, [consulta: 2 de Marzo 1998].

REACCIUN. "Página Principal", <http:/wwww. reacciun.ves, [consulta: 17 de Febrero 1998].

Schoffstall, M. (1991, April). Foundations and history of the Intemet and PSINet en Greller, Leonore M. (1993). "Groupware and Interpersonal Text: The Computer as a Medium of Communication".

Silvio, José F. (1998) "La Virtualización de las Universidades". Ponencia presentada en el Congreso Visionarios 98, Caracas, Abril 22-24. Universidad Católica Andrés Bello. "Página Principal". <http://www ucab.edu.ve/UCAB/Fa cultades/Ingenieria (Idem) / Derechos, [consulta: 5 de Marzo de 1998].

Universidad Central de Venezuela. "Sistema Gerencial de Apoyo a la Información. Rectorado". <http://www.cosmopoima.ing.ucv.ve>;

http://ceea.arq.ucv.ve; http:/hierra.ciencs.ucv.ve; Informática http://rww.rest.ucv.ve; Imágenes biomédicas http://wnw.caib- co.ucv.ve;

http://www.ing.ucv.ve/matemáti-

ca/index.htm;

http://www.ucv.ve.ffacjupol/derecho/derecho.html. (15 de Febrero 1998).

Universidad de Carabobo. "Fundación Centro de Información y Documentación". 9 de Diciembre 1996.

http://www.cid.ve; http://wnw.tacesuc.org.ve y http://www.postgrado.cid.ve

Universidad Centro Occidental Lisandro Alvarado. http://redisa.ucla.edu.ve. (7 de Marzo 1998).

Universidad de los Andes. "Servicio de Documentación de la Universidad de Los Andes (SeDULA)". 28-01-98. http://wnw.ula.ve; http://wrww.ing.ula.ve; http://ciens.ula.ve; forest.ula.ve; pgcomp.ula.ve; forest.ula.vel wleon/ (15 de Febrero 1998).

Universidad de los Llanos Ezequiel Zamora. "Enlace. Pagina Informativa de la Oticina de Enlace". Enero 1998. http:/www.unellez,edu.ve (9 de Marzo 1998).

Universidad de Nueva Esparta. "Página Principal". (http://www.une.edu.ve), (5 de Marzo 1998). Páginas particulares diseño, 80/ electrónico 80/ turismo; administración; computación; civil.

Universidad de Oriente. "Universidad de Oriente". http://www,mochima.sucre.udo.edu.ve (6 de Marzo 1998).

Universidad del Zulia. (1994), Filosofía de Gestión de la Univer sidad del Zulia. 32p. Maracaibo. Universidad del Zulla. http://urww.luz.ve/ceela; http://www.licom.ciens.luz.ve.

Universidad Metropolitana. "Página Principal", (http://www.etheron.net/ethe- 
ron/jaz/unimet.unimet.htm), (5 de Marzo 1998).

\section{Universidad}

Nacional

Abierta.

http://www.memberes.Tripod.com/ maguicha/postgrado.html.

Universidad Nacional Experimental del Táchira. "Centro de Estudios de Teleinformática". 20 de Octubre 1997. http:/www.unet.ve/ ceti/. (9 de Marzo 1998).

Universidad Nacional Experimental Francisco de Miranda. "Universidad Nacional Experimental Francisco de Miranda". http://www.unefm.edu.ve (6 de Marzo 1998).

Universidad Nacional Experimental Politécnica Antonio José de Sucre. "Universidad Nacional Experimental Politécnica Antonio José de Sucre". http://www.unexpo.edu.ve (6 de Marzo 1998).

Universidad Nacional Experimental Simón Rodriguez. "Interacción de la Universidad con su Entorno". 1997. http://www.syr.edu/-jmontoya/unesrl. (7 de Marzo 1998).

http://www.syr.edu/ jmontoya/unesrl. (24 de Febrero 1998).

"Preparándonos para la Sociedad de la Información". 1997. http:/www.syr.edu/-jmontoyalunesrl. (7 de Marzo 1998).
Universidad Rafael Belloso Chacin. http://www.telcel.nel.ve/urbel

Universidad Rafael Maria Baralt. "Programa de Investigación". (http://www.civila.com/archivos/venezuela), (3 de Marzo 1998).

Universidad Rafael Urdaneta. http://www.uru.edu

Universidad Simón Bolívar. "La Dirección de la Gestión de la Información". http://www.usb.ve; http://159.90.32.137 inolex.htm (Ingenieria Eléctrica); http://www.ldc.usb.ve/(Lab. Computación); http://159.90.166.205 (Lab. Electrónica). (15 de Febrero 1998).

(si). Siatema de Información de la Universidad Simón Bolivar. Conceptualización y Plan para su implementación y desarrollo (Fase 1990-1993). 12p. Caracas.

. "Sistema de Información de la Universidad Simón Bolivar (SI-USB)". http://www.usb.ve (15 de Febrero 1998).

Universidad Tecnológica del Centro. http:/hwww.unitec.edu.ve/ (7 de Marzo 1998).

Villalobos, Orlando (1998). Política y Gerencia de la Comunicación Social en la Universidad del Zulia. Tesis de Maestría. Universidad del Zulia. 